\title{
Tecnologías de la web semántica para el desarrollo de una memoria organizacional en la atención de usuarios en un departamento de tecnología
}

\section{Semantic web technologies for the development of an organizational memory to support users in a technology department}

DOI: $10.46814 /$ lajdv3n2-016

Recebimento dos originais: 23/12/2020

Aceitação para publicação: 26/02/2021

\author{
Alonso Perez-Soltero \\ Doctor en Tecnologías de la Información y las Comunicaciones \\ Universidad de Sonora; Blvd. Luis Encinas y Rosales, Hermosillo Sonora, México \\ E-mail: aperez@industrial.uson.mx \\ Miguel Enrique Lopez-Muñoz \\ Maestro en Ingeniería Industrial \\ Universidad de Sonora, Blvd. Luis Encinas y Rosales, Hermosillo Sonora, México \\ E-mail: miguel.lopez@unison.mx \\ Mario Barcelo Valenzuela \\ Doctor en Tecnologías de la Información y las Comunicaciones \\ Universidad de Sonora; Blvd. Luis Encinas y Rosales, Hermosillo Sonora, México \\ E-mail: mbarcelo@industrial.uson.mx
}

\section{RESUMEN}

El objetivo del presente artículo es proponer un modelo de memoria organizacional basado en tecnologías de la Web semántica, para gestionar el conocimiento originado por la atención a usuarios de un departamento de tecnología de una Institución de Educación Superior en el Noroeste de México. El modelo propuesto representa de manera global las actividades de atención de las diversas áreas del departamento y propone una visión conceptual para el desarrollo de una memoria organizacional que favorezca la gestión de dicho conocimiento. Esta memoria, incluye la organización, almacenamiento y recuperación del conocimiento generado por los técnicos al atender las órdenes de servicio, la interacción con los usuarios y dispositivos, además del que obtienen de las capacitaciones recibidas. Lo anterior contando con una plataforma soportada en tecnologías de la web semántica.

Palabras Clave: Gestión del conocimiento, memoria organizacional, Web Semántica, departamento de tecnología.

\section{ABSTRACT}

The aim of this paper is to propose a model of organizational memory based on semantic Web technologies, for managing knowledge in the areas of technical support and technology infrastructure from the IT department of an institution of higher education in northwest Mexico. The proposed model represents a comprehensive manner the existing knowledge in the areas of technical support and networking and telecommunications from the IT department and proposes a conceptual vision for the development of an organizational memory that favors such knowledge management. This memory, it includes the organization, storage and recovery of the knowledge generated by the technical personnel on having attended to the orders of service, the interaction with the users and devices, besides that they obtain of the received trainings. The previous aspects possessing a platform supported by semantic web technologies. 
Keywords: Knowledge management, organizational memory, semantic Web, technology department.

\section{INTRODUCCIÓN}

Actualmente, las organizaciones que buscan estar en mejores condiciones competitivas en el mercado se encuentran en continuos procesos de cambio internos para responder al entorno donde se desarrollan. Estos procesos también se viven dentro de las instituciones educativas con el afán de ofrecer los mejores servicios a estudiantes, personal académico y administrativo. Sin duda, y al igual como sucede en otro tipo de organizaciones, las áreas de tecnologías de información tienen una función muy importante que es la de proveer servicios de calidad a todos sus usuarios.

Uno de los factores presentes en las organizaciones y fundamental en las áreas de tecnologías de información, es el conocimiento que poseen las personas que participan en ellas. Es clave contar con estrategias organizacionales y tecnológicas que faciliten la manera de gestionar dicho conocimiento.

El objetivo del presente artículo es proponer un modelo de memoria organizacional basado en tecnologías de la Web semántica y colaboración en tiempo real para gestionar el conocimiento de las áreas de soporte técnico e infraestructura tecnológica del departamento de tecnología de una Institución de Educación Superior en el Noroeste de México.

La estructura del artículo inicia con los antecedentes y problemática, después se presenta el marco teórico con la descripción de los principales conceptos como gestión del conocimiento, memoria organizacional y Web semántica. Luego se propone un modelo de memoria organizacional basado en tecnologías de la Web semántica para gestionar el conocimiento de las áreas de informática de una Institución educativa, posteriormente, se hace una descripción de la validación del modelo propuesto, finalmente, se muestran las conclusiones del presente trabajo.

\section{ANTECEDENTES Y PROBLEMÁTICA}

El presente proyecto se desarrolla en una institución de educación superior ubicada en el noroeste de México, particularmente en su departamento de tecnología.

Este departamento de tecnología, cuenta con 5 áreas fundamentales para brindar los servicios básicos de informática que son: Atención a usuarios (mesa de ayuda) que es el área encargada de darle seguimiento y atención a los usuarios académicos y administrativos de la institución, esta área tiene la función de asignar las órdenes de servicio a las diferentes áreas dependiendo del tipo de servicio y de otros factores como prioridades, tipo de información, usuarios, entre otros; Desarrollo Web que es el área encargada del desarrollo y diseño de los portales web de la institución (Página institucional, portales departamentales, por mencionar algunos) y además brinda servicio de soporte a los usuarios externos que contratan servicios tecnológicos proveídos por el departamento; Desarrollo que es el área encargada de analizar, desarrollar e implementar los sistemas administrativos que utilizan los diversos departamentos 
de la institución para llevar el control de costos, actividades, personal, alumnos, entre otros; Soporte Técnico, área encargada de seleccionar, instalar y dar mantenimiento preventivo y correctivo a equipo de cómputo de la institución, además es la encargada de administrar el sistema de antivirus, configuración de correo electrónico y dar soporte a usuarios en casos de respaldo de información y configuración de software; Infraestructura tecnológica, esta área encargada de administrar todo el contenido de la red institucional, además de administrar y configurar los accesos y privilegios de los usuarios de la red, también se encarga de administrar y configurar los servicios administrados y dar soporte a los usuarios académicos y administrativos para solucionar problemas de conectividad, respaldo de información, instalación y configuración de servidores.

En el departamento de tecnología las áreas de infraestructura tecnológica y soporte técnico están conjuntamente ligadas, ya que realizan algunas actividades en común como son la configuración de cuentas de correo electrónico, configuración de software y respaldo de información. El área de atención a usuarios asigna estas actividades a cada área dependiendo de varios factores como prioridades, niveles de usuario y el nivel de complejidad y confidencialidad de la información a gestionar, estas actividades son canalizadas a las áreas en forma de órdenes de servicio, estas últimas son notificaciones que llegan en forma de notificación electrónica y/o correo electrónico donde se indica a qué técnico se ha asignado la orden, quién es el usuario solicitante, la descripción de la solicitud, así como los datos de contacto del solicitante. Cuando el técnico termina de dar servicio a la orden, la finaliza electrónicamente dejando así un historial que solo contiene información de fecha de inicio, descripción y fecha de terminación.

La problemática que se presenta es en varias vertientes. Una de ellas, es cuando el área de atención a usuarios recibe una orden de servicio para solucionar problemas de accesos al correo electrónico, instalación y configuración de software, problemas de acceso a la red y migración o respaldos de equipo de cómputo o software; los puede canalizar tanto a personal área de soporte técnico como a personal del área de infraestructura tecnológica dependiendo de la urgencia de la solicitud, la confidencialidad de la información utilizada o la persona que lo esté solicitando. Aunque el objetivo final de la actividad solicitada puede ser el mismo, los métodos y técnicas para solucionarlo pueden variar dependiendo del técnico que lo esté atendiendo, debido a que no existen procedimientos debidamente estandarizados. Uno de los principales problemas que se generan a través de este esquema de canalización, es que el técnico que soluciona la orden de servicio se puede encontrar con varias dificultades técnicas que ocasionan que este tenga que realizar modificaciones al procedimiento establecido en la solución de dichos problemas y llevar a cabo un nuevo procedimiento o una nueva técnica para solucionarlo. En la mayoría de las ocasiones, el conocimiento generado a través de este tipo de relaciones con los usuarios y equipo se queda solamente en la experiencia del técnico y muchas veces puede ser olvidada por la persona que la generó.

Otra situación que se vive por parte del personal, ya sea del área de soporte técnico como el de infraestructura tecnológica, es que al acudir a atender las órdenes de servicio no se cuenta con historial 
de los procedimientos utilizados en los equipos como los permisos y privilegios que tenga el usuario y exista desinformación del tratamiento que se deberá de dar a los usuarios y al equipo, generando así que el técnico opte por eliminar todo el trabajo de configuración y empezar a trabajar desde el principio para así tener una noción del trabajo realizado.

Otro problema observado es en la parte de capacitación. El departamento de tecnologías invierte cantidades considerables de dinero en capacitación para sus técnicos, pero muchas veces debido a limitaciones financieras, la capacitación se le ofrece a un grupo limitado de personas y en ocasiones hasta a una sola persona, pero la mayoría de las ocasiones esta información obtenida de la capacitación de limita a la persona que la adquirió y no se comparte a las personas interesadas o las personas que deberían de conocer esa información por medio de algún programa de divulgación informática o algún otro implementado por la institución. El desconocimiento o la falta de promoción y divulgación del nuevo conocimiento generado u obtenido por el personal de las áreas mencionadas a través de cursos y experiencia propia genera a el departamento un gasto considerable de recursos. Todo lo anterior ocasiona que el tiempo de atención a los usuarios y el tiempo de capacitación de los técnicos en algunas ocasiones no sea óptimo.

En pocas palabras, se puede conceptualizar el problema dentro de las áreas de infraestructura tecnológica y soporte técnico del departamento de tecnología de esta institución educativa como la inadecuada retención, recuperación, compartición y distribución del conocimiento generado por los técnicos en sus actividades de seguimiento a las órdenes de servicio por concepto de soporte técnico a equipos de cómputo y servicios de red emitidas por el personal académico y/o administrativo de la Institución, para ser utilizado y aprovechado por los integrantes de la mencionada dirección para ofrecer un mejor servicio a los usuarios.

\section{CONCEPTOS TEÓRICOS Y EXPERIENCIAS SIMILARES}

A continuación, se abordarán los conceptos de gestión del conocimiento, memoria organizacional y web semántica, ya que se consideran tres conceptos de la fundamentación teórica de este trabajo y en las que sirven como las bases para el modelo de memoria organizacional que se propone. También se incluyen algunos casos y experiencias recientes que tienen relación con este proyecto.

\subsection{GESTIÓN DEL CONOCIMIENTO}

Dentro de una organización existen numerosas fuentes de conocimiento que muchas veces no son controladas y/o gestionadas ocasionando así que este conocimiento generado no sea utilizado en beneficio de ella, el conocimiento es un recurso que todas las organizaciones poseen y que es especialmente interesante, ya que se puede almacenar, utilizar, movilizar, desarrollar, es decir, gestionar de diferentes formas (Hernandez y León, 2009). 
Según Dalkir (2017) la gestión del conocimiento presenta un enfoque deliberado y sistemático para asegurar la completa utilización del conocimiento de una organización, el potencial de las destrezas individuales, las competencias, los pensamientos, las innovaciones y las ideas para crear una organización más eficiente y efectiva. De acuerdo con Gonzalez et al. (2004) la gestión del conocimiento puede ser entendida como la planificación, coordinación y control de las actividades que lleven a la creación, captura y transmisión del conocimiento en forma efectiva.

La gestión del conocimiento ha surgido como la estrategia, que las entidades necesitan adoptar para gestionar y utilizar el conocimiento organizacional (Rodríguez y Dante, 2008). Esto permite que la organización obtenga beneficios del conocimiento generado en su entorno y así este pueda contribuir con el desempeño de la empresa minimizando costos de capacitación y haciendo más eficientes y eficaces un número considerables de procesos laborales.

\subsection{MEMORIA ORGANIZACIONAL}

Este concepto ha sido tratado por diversos autores en los últimos años. Uno de ellos, Stein (1995) dice que los conocimientos y características de una organización que quieran ser transmitidas del pasado a los futuros miembros del sistema social de la empresa, solamente van a ser posibles si se tiene la memoria organizacional. El conocimiento es el capital clave de la organización. La memoria organizacional extiende y amplifica este capital con la captura, organización, diseminación y reuso del conocimiento creado por sus empleados. Sin embargo, la memoria organizacional no es sólo un facilitador de la acumulación y preservación del conocimiento, sino que también permite compartirlo con los demás miembros de la organización (Ackerman e Halverson, 2000).

De acuerdo con el enfoque y orientación de este trabajo, interesa retomar la definición propuesta por Perez-Soltero (2006). En este sentido, se conceptualiza la memoria organizacional como el lugar donde se almacena el conocimiento de una organización generado en el pasado para utilizarlo de forma inteligente y racional en el presente y en el futuro, con la característica de que este repositorio sea fácil de acceder por los miembros de la organización involucrados e interesados en aprovechar dicho conocimiento. Otro punto relevante planteado por Conklin (1997) es que la memoria organizacional involucra la codificación de la información a través de una representación adecuada, la cual posteriormente tendrá un efecto sobre la organización en el momento en que sus miembros interpreten la información almacenada a la luz de las condiciones actuales y futuras de la organización.

\subsection{WEB SEMÁNTICA}

El concepto de Web semántica ha recibido múltiples definiciones a lo largo de la historia. BernersLee et al. (2001) comentan que la Web semántica no se encuentra separada de la Web actual, sino que es una extensión de ella, incluyendo una estructura que permite a los contenidos de las páginas web 
expresarse de una manera que pueda ser comprensible por los dispositivos informáticos y que posibilite el trabajo en cooperación usuario-ordenador.

Hoy en día la utilización de la Web semántica para manejar contenidos de información es gran artefacto para proveer soluciones de compartición, localización, integración y la reutilización de conocimiento a través de la implementación de diversas herramientas tecnológicas que facilitan estos procesos de una manera práctica, basados en estándares previamente establecidos (Hitzler, 2020)

A través de la Web semántica han surgido nuevas tecnologías estándares que la sustentan como son el XML, XML Schema, RDF / RDFS, OWl, entre otras. En general, la web semántica ofrece a la Web tradicional una plataforma más apropiada para la integración de información basada en que los datos tienen significados bien definido además de que el software en lugar de seres humanos, puede utilizar gran variedad de fuentes y generar información para mejorar la adquisición, el almacenamiento y la recuperación de conocimientos significativo de la organización (Barcelo-Valenzuela et al., 2006).

\subsection{ALGUNOS ESTUDIOS Y EXPERIENCIAS RELACIONADAS}

A continuación, se describen algunos estudios y/o experiencias recientes relacionadas a este proyecto.

Uno de ellos descrito por Hernández y León (2009) donde hace referencia a que el conocimiento generado en esas empresas no se documenta y en caso de hacerlo es almacenado en memorándums, manuales y archivos de texto electrónico. Comenta que esta forma de transferir ese conocimiento genera a las empresas grandes pérdidas de tiempo y alta inversión en recursos humanos y financieros, principalmente cuando se quiere transmitir a alguna otra dependencia o persona. En su documento se propone realizar e implementar un sistema de memoria organizacional, ya que la implementación de un sistema de este tipo en la empresa traerá beneficios como almacenar el conocimiento generado por los individuos para un uso posterior, facilitar el aprendizaje organizacional, contribuir a la eficiencia y la efectividad en la toma de decisiones, la información estará disponible oportunamente, entre otros. De igual manera propone un sistema que constituye una capa que transforma la información desordenada y dispersa proveniente de los procesos en conocimiento disponible a la empresa utilizando tecnologías de workflow.

Otro estudio relacionado a este proyecto es el de Kei-Hoi y Huajun (2010) donde mencionan que los trabajos científicos para investigar la medicina china y la medicina occidental han estado generando una gran cantidad de datos para ser compartidos en un entorno de estándares globales, debido a esto, se proporciona una visión general del uso de la Web semántica e identifica tecnologías representativas de su aplicación en la medicina china, además de proponer a la misma como un estándar para representar los datos de la medicina china y facilitar su integración con los datos de la medicina occidental. Propone el uso de formatos OWL/RDF para la representación de los datos en los sistemas Web semánticos. 
Por su parte, Weinberger et al., (2008) presentan una propuesta para evaluar a las memorias organizacionales basadas en ontologías llamada KnowledgeEco, esta metodología sería utilizada para implementar una memoria organizacional dentro de una organización ya evaluada por los conceptos de una ontología, señalando cuáles elementos de la ontología no se encuentran dentro de la memoria organizacional, además de aplicar varias reglas o normas que ayudarán a evaluar el impacto dentro de la organización. Los autores presentan un caso de estudio en donde prueban la viabilidad del sistema KnowledgeEco dentro de tres diferentes ambientes. Una entidad de servicios de salud en Israel, una organización dedicada a la ingeniería civil y un instituto de investigación en Alemania. Dentro de estos tres casos de estudio se descubre que la mayor parte de estas entidades tienen problemas para la compartición de conocimiento, además de una pobre participación de las personas. Al implementar esta metodología dentro de estas organizaciones, los autores concluyen que esta alienta a las personas a utilizar las herramientas y a compartir su conocimiento, además de que KnowlwdgeEco se puede utilizar como un modelo de referencia para describir qué es lo que existe y qué es lo que falta dentro de la organización, además de que facilita el aprendizaje organizacional como fuente de mejores prácticas de los procesos.

Otro caso que utiliza las tecnologías de la Web semántica, específicamente para la implementación de una memoria organizacional, es el presentado por Khilwani y Harding (2017), en el que describen un marco de trabajo en donde integran diversas tecnologías de la web semántica como XML, RDF, OWL entre otras para construir modelos de consulta de información basados en esquemas que dividen a los datos en 4 áreas específicas: Información general, Información técnica, Información capturada y conocimiento refinado. El sistema implementado por los autores para construir la memoria organizacional contempla cinco áreas: Procesamiento de documentos, análisis de texto, generación de RDF, revisión de RDF y análisis y filtrado. El objetivo principal de esta aplicación es que a través de las áreas previamente descritas, se construye una memoria corporativa, generando un catálogo relacional de toda la información generada por los procesos administrativos.

\section{PROPUESTA DE UN MODELO DE MEMORIA ORGANIZACIONAL}

De acuerdo con la información revisada previamente y a la problemática planteada dentro de la Dirección de Informática, en la figura 1 se propone el diseño de una memoria organizacional basada en tecnologías de la Web semántica. 


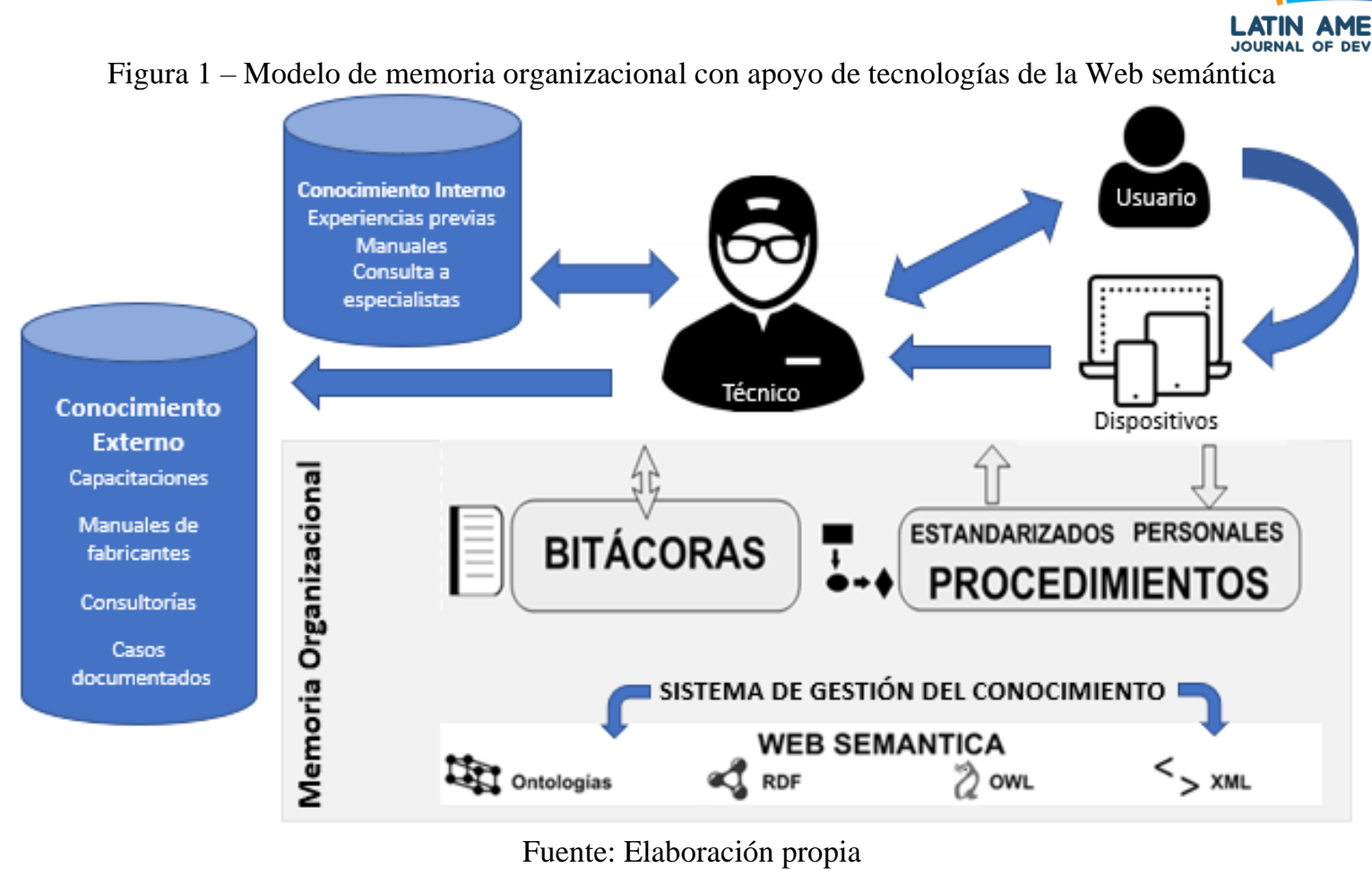

El objetivo de este modelo es esquematizar de manera global cómo se presenta el conocimiento en las áreas de soporte técnico e infraestructura tecnológica del departamento de tecnología y proponer una visión conceptual para el desarrollo de una memoria organizacional que favorezca la gestión de dicho conocimiento. Este modelo contempla aspectos tanto organizacionales como tecnológicos.

Desde el punto de vista organizacional, es importante considerar la participación y el compromiso del director y los responsables de las áreas involucradas. Además, se requiere la colaboración de todos y cada uno de los técnicos que participan. Es importante hacer una evaluación y revisión de sus métodos de trabajo para que puedan orientarse hacia un enfoque de la gestión del conocimiento. En otras palabras, es fundamental el compromiso de la dirección, cambio de cultura organizacional, capacitación, actualización de las formas de trabajo y colaboración de todos los involucrados.

Desde el punto de vista tecnológico, es importante contar con una plataforma tecnológica que facilite la retención, recuperación, compartición y distribución del conocimiento generado dentro de estas áreas. Para esto, la utilización de tecnologías de la Web semántica facilitará la organización, almacenamiento y recuperación del conocimiento generado por los técnicos al atender las órdenes de servicio, la interacción con los usuarios y dispositivos, además del que obtiene de las capacitaciones recibidas.

Considerando lo anterior, se concibe a este modelo de memoria organizacional como un enfoque integral de trabajo orientado hacia el aprovechamiento del conocimiento existente dentro de un departamento de servicios tecnológicos, en particular en las áreas de soporte técnico e infraestructura tecnológica. En otras palabras, constituye un apoyo fundamental para gestionar adecuadamente el conocimiento presente y futuro en las mencionadas áreas. 
A continuación, se hace una explicación detallada de los elementos más importantes del modelo y la interacción que existe entre ellos.

\subsection{ELEMENTOS}

Los elementos que contiene el modelo de memoria organizacional propuesto son técnicos, usuarios, dispositivos, bitácoras, procedimientos y conocimiento externo los cuales se describen a continuación.

Técnicos: Recurso humano de la Dirección de Informática que se encarga de dar seguimiento a las órdenes de servicio con concepto de soporte técnico a equipos de cómputo y servicios de red emitidas por el personal académico y administrativo de la Institución, tienen la capacidad de administrar la información de inicio de sesión de los usuarios a los servicios de red institucional y configurar los dispositivos por medio de los cuales se hará la conexión.

Usuarios: Personal de la institución que hace uso de las instalaciones de red y de los dispositivos informáticos, cuenta con información como su cuenta de inicio de sesión a los dispositivos y a la infraestructura de red, también tiene la capacidad de emitir solicitudes de servicio al departamento de tecnología, las cuales serán canalizadas a las diversas áreas internas.

Dispositivos: Equipos Informáticos como computadoras de escritorio, laptops, tablets pc, dispositivos móviles, entre otros; que se utilizan para realizar las actividades diarias de la institución y utilizan la red universitaria para conectarse a internet, la mayoría de estos dispositivos está unida al dominio de red institucional y se ajusta a las directivas de seguridad establecidas.

Bitácoras: Información generada a partir de los seguimientos de órdenes de servicio por parte de los técnicos donde se almacena información como problemática vista, procedimientos utilizados y sugerencias.

Procedimientos: Se dividen en dos partes, procedimientos personalizados que son una secuencia de pasos propuestos por algún técnico que pueden dar solución a alguna problemática observada, pero que aún no han sido aprobados oficialmente por el departamento, aunque de igual manera son documentados; y los procedimientos estandarizados que son los que ya han sido revisados por la dirección y han sido aprobados como procedimientos oficiales que cumplen con las normas de seguridad establecidas.

Conocimiento externo: Procedimientos y técnicas compartidas y sugeridas por los mismos proveedores de hardware y software, personas que laboran en instituciones externas con casos similares, capacitaciones impartidas y consultores externos.

\subsection{INTERACCIÓN ENTRE LOS ELEMENTOS}

El proceso de seguimiento a una orden de servicio por parte de las áreas de soporte técnico e 
infraestructura tecnológica comienza cuando el usuario realiza una orden de servicio a través del área de atención a usuarios, orden que es canalizada a un técnico de alguna de las dos áreas dependiendo de la problemática presentada. Cuando algún técnico de estas áreas recibe la orden de servicio, procede a ponerse en contacto con el usuario que experimenta la problemática y se dispone a revisar si esta es causada por algún error en los servicios de red o si es algún error causado por alguna mala configuración del dispositivo informático que está siendo utilizado, además de revisar si existe algún procedimiento establecido para solucionar esta problemática y si no es así, puede proponer algún procedimiento y/o revisar si existe algún otro procedimiento propuesto por alguna consultoría externa o a algunas empresas fabricantes de hardware o software involucradas. Después de realizar los procedimientos el técnico almacena el conocimiento generado en una bitácora de trabajo la cual podrá ser consultada cuando sea necesario por el mismo técnico o algún otro que lo requiera.

Toda esta información generada a través de estas actividades estará almacenada y será administrada por un sistema basado en tecnologías de web semántica como ontologías, XML, RDF, OWL, entre otras, que podrá ser consultado por los técnicos a través de los dispositivos informáticos que tiene a su disposición y se encuentren conectados a la red institucional.

\subsection{BENEFICIOS DE LA MEMORIA ORGANIZACIONAL}

De acuerdo con el enfoque planteado en este modelo para organizar y almacenar el conocimiento generado por los técnicos al resolver órdenes de servicio y el generado a través de las capacitaciones recibidas; se pretende obtener diferentes beneficios para la Dirección de Informática entre los que se encuentran:

Seguimiento y control de usuarios: Al contar con un historial de bitácoras y procedimientos para cada usuario en donde se almacenen las diferentes problemáticas que han experimentado y la manera en que estas han sido resueltas, los técnicos del departamento podrán solucionar los problemas que se presenten de una manera más eficiente, ya que si estos se han presentado con anterioridad, se dispondrá de una serie de procedimientos con los cuales se podrán solucionar sin tener que realizar una búsqueda exhaustiva de soluciones. Además, se tendrá la ventaja de que cualquier técnico que acuda a darle seguimiento a la orden de servicio del usuario, podrá conocer cuáles han sido las actividades que fueron realizadas anteriormente por algún otro técnico.

Seguimiento y control de dispositivos: Al contar con una bitácora de configuraciones de hardware y software de los dispositivos informáticos utilizados en la institución, se podrá reducir el tiempo de atención, ya que el técnico contará con la relación de procedimientos que han sido implementados en el dispositivo y podrá tener una visión más coherente del o los procedimientos que deberá seguir para solucionar la problemática presentada.

Seguimiento y control de los técnicos: El llevar un seguimiento y control de las actividades 
realizadas por los técnicos, como órdenes de servicio terminadas y procedimientos utilizados y propuestos, brindará al departamento de tecnología una plataforma ideal para evaluar la productividad de estos, generando así información valiosa para diseñar programas de capacitación y desarrollar programas de trabajo acordes a las actividades presentadas en la información del sistema.

Seguimiento y control de bitácoras: Al contar con una bitácora de actividades relacionadas a cada usuario, dispositivo y técnico, se podrá disminuir considerablemente el tiempo de atención y se podrá hacer más eficaz el servicio, ya que de inmediato se contará con información útil donde se incluyen tiempos de servicio a usuarios, conocer las relaciones técnicos-usuarios, qué procedimientos se utilizaron para resolver alguna problemática, qué procedimientos fueron propuestos por algún técnicos, horarios en los que las solicitudes fueron atendidas, problemáticas observadas, comentarios por parte de los técnicos y los usuarios, por mencionar algunas.

Seguimiento y control de procedimientos: El contar con documentación de los procedimientos utilizados para resolver problemas específicos de usuarios y dispositivos, será de gran ayuda para los técnicos en caso de enfrentarse a alguna problemática diferente a la que se hayan enfrentado anteriormente, ya que el tiempo de documentación y búsqueda acerca de esa problemática se reducirá en gran medida al tener acceso al sistema donde se encuentren almacenados todos los procedimientos utilizados y propuestos por algunos otros técnicos que hayan experimentado con esa problemática en algún otro momento.

\section{VALIDACIÓN DEL MODELO PROPUESTO}

La implementación del modelo incluirá el desarrollo de una metodología que permita llevarlo a la práctica, que contemple los aspectos organizacionales como tecnológicos.

Por su parte, desde el punto de vista organizacional se tendría que desarrollar una estrategia en donde se exhorte a las áreas relacionadas el uso de la plataforma y se expliquen los beneficios que se tendrán con su utilización, además, la dirección del departamento tendrá que nombrar a un comité de evaluación de procedimientos, los cuales tendrán la cualidad de revisar los que han sido propuestos por los técnicos a través de su experiencia en el campo de trabajo y decidir si cumplen o no con las normas de seguridad establecidas, para posteriormente estandarizarlos y nombrarlos como procedimientos oficiales.

En cuanto al aspecto tecnológico, se desarrollará un portal web que utilizará tecnologías de la web semántica para organizar y almacenar el conocimiento generado por los técnicos al resolver órdenes de servicio, la interacción con los usuarios y dispositivos, además del que se obtiene de las capacitaciones recibidas. Se podrá acceder a él a través de los diversos dispositivos informáticos que se encuentren conectados a la red institucional.

Este portal dispondrá de un módulo de consulta vía Web, para permitir su acceso durante todo el 
proceso de recepción y atención a las órdenes de servicio, por parte de todos los involucrados en el proceso de auditoría. También se incluirá la capacidad para la generación automática de formularios e informes para automatizar la documentación de los procesos y la actualización de las bitácoras y una interfaz donde se muestre en un formato entendible la información requerida.

\section{CONCLUSIONES}

El presente artículo muestra la propuesta de un modelo de memoria organizacional basado en tecnologías de la Web semántica. Este modelo se está desarrollando para ser validado dentro del departamento de tecnología que ofrezca servicios a usuarios administrativos y académicos en una Institución de Educación Superior del noroeste de México. Este modelo incluye los elementos más importantes y la relación entre ellos, además, describir puntos importantes para su implementación desde el punto de vista organizacional y tecnológico.

La implementación de un modelo de memoria organizacional como el aquí propuesto traería muchos beneficios que van desde la reducción del tiempo de asistencia técnica a los usuarios, llevar un control cronológico y conceptual de la información generada evitando redundancia en las actividades diarias del personal, incrementar la generación de nuevas técnicas y métodos que hagan las actividades sean más eficientes y eficaces, entre otras ventajas. La implementación de esta memoria organizacional con apoyo de tecnologías de la Web semántica es muy conveniente, ya que facilitaría organizar la información por medio de conceptos y relaciones haciendo más eficiente la ordenación y localización de esta. 


\section{BIBLIOGRAFÍA}

ACKERMAN, M.; HALVERSON, C. Re-examining organizational memory. Communications of the ACM, 43(1): 58-63, 2000.

BARCELO-VALENZUELA, M.; SANCHEZ-SCHMITZ G. G.; PEREZ-SOLTERO A. La Web Semántica como apoyo a la Gestión del Conocimiento y al Modelado Organizacional. In: Revista Ingeniería Informática, Chile, 2006.

BERNERS-LEE, T.; HENDLER, J.; LASSILA, O. The Semantic Web: A new form of Web content that is meaningful to computers will unleash a revolution of new possibilities, Scientific American.com, 2001 .

CONKLIN, E.J. Designing Organizational Memory: Preserving Intellectual Assets in a Knowledge Economy, 1997. http://cognexus.org/dom.pdf

DALKIR, K. Knowledge Management in Theory and Practice. (Third Edition). MIT Press, 2017.

GONZÁLEZ, A. L.; CASTRO J. P.; RONCALLO M. Diagnóstico de la gestión de conocimiento en una empresa grande de Barranquilla (Colombia): Una actividad de vinculación cooperativa universidad sector productivo, Ingeniería \& Desarrollo, 16: 70-103, 2004.

HERNANDEZ, M. A. A.; LEÓN, D. O. W. S. Modelo de memoria organizacional soportado en "Workflow" en empresas comercializadoras mixtas. In: XI Taller Internacional de Gestión Tecnológica e Innovación y su Aplicación en las Organizaciones - GESTEC’2009, 2009.

HITZLER, P. A review of the semantic web field. Communications of the ACM, 64(2), 76-83, 2021. doi: $10.1145 / 3397512$

KEI-HOI, C.; HUAJUN, C. Semantic Web for data harmonization in Chinese medicine. Chinese Medicine, 5: 1-5, 2010.

KHILWANI, N., HARDING, J.A. Managing corporate memory on the semantic web. Journal of Intelligent Manufacturing 27: 101-118, 2016. https://doi.org/10.1007/s10845-013-0865-4

PEREZ-SOLTERO, A. El papel de las Tecnologías de Información y la Memoria Organizacional dentro de las Organizaciones Inteligentes. Novática, No. 182: 52-55, 2006.

RODRÍGUEZ, I. M. A.; DANTE, G. P. La Segunda Generación de la Gestión del Conocimiento: un nuevo enfoque de la gestión del conocimiento. (Spanish). Ciencias de la Información, 39(1): 19-30, 2008.

STEIN, E.W. Organizational Memory: Review of Concepts and Recommendations for Management, International Journal of Information Management, 15(1): 17-32, 1995.

UNIVERSIDAD DE SONORA, D. I. Antecedentes de la Dirección de Informática de la Universidad de Sonora. v. 2010. n. 19 de Diciembre. Hermosillo Sonora 2010.

WEINBERGER, H.; TE'ENI, D.; FRANK, A. J. Ontology-based evaluation of organizational memory. Journal of the American Society for Information Science and Technology, 59(9): 1454-1468, 2008 